\title{
Intimate Partner Violence in Muslim Communities
}

\section{Dheeshana Jayasundara ${ }^{1}$, Randall Nedegaard ${ }^{1}$, Bonita Sharma ${ }^{2}$ and Kenneth Flanagan ${ }^{1}$}

${ }^{1}$ University of North Dakota, Grand Forks, ND, USA

${ }^{2}$ University of Texas, Arlington, TX, USA

\begin{abstract}
Despite intimate partner violence (IPV) being a global phenomenon, there is a scarcity of literature on the IPV in Muslim communities. This is especially true when it comes to empirical studies. As a first step towards remedying this deficit, this paper conducted a systematic review of the current empirical literature pertaining to IPV among Muslims. The purpose of the review is to develop an overview of the findings of the past studies that empirically examine the topic of IPV and Muslims. This overview assesses the areas or topics in the existing literature, critiques the methodologies used, documents their results, and identifies the gaps for future research. The final analysis includes 35 empirical studies on this topic both in the US and globally. The findings are discussed under the following identified themes: prevalence and types of abuse, factors associated with exposure to IPV, attitudes towards IPV, experiences of coping and support, IPV and health consequences, and service response. Policy and future research implications of this study are presented.For instance, future research needs to focus on the intersections of religion, culture, political history, socioeconomic status, immigration status and the history of patriarchal oppression related to cultural practices of families and their effects on IPV.
\end{abstract}

Keywords: Muslim; Intimate partner violence; Religion; Islam

\section{Introduciton}

Intimate Partner violence (IPV) against women is a problem that unfortunately plagues all societies. The World Health Organization [1] survey estimates that as many as $69 \%$ of women in the world can be impacted by IPV. It can have significant psychological, physical, social, economic, and spiritual implications that can negatively affect future generations, social relationships, and productive capacity [2,3]. Many factors intersect with situations of partner violence to either enhance survivor resilience or to further complicate IPV situations [4,5].

Religion is one of the factors that play an especially important role when it comes to situations of IPV. When studying IPV it is important understand the interplay between IPV and the multiple dimensional roles religion plays while intersecting with other dimensions of culture. No religion condones IPV and this behavior disrespects religious teachings that promote and peace and harmony. Yet many IPV perpetrators use religion as another weapon of abuse. As Marie Fortune [6], one of the pioneering faith leaders acting against domestic violence said:

...abusers ...misuse and distort scripture to justify their choice to harm another person because they have power over that person. It is very easy to misuse and distort sacred texts. All you have to do is to lift something out of context with no understanding or appreciation of its history and meaning and use it to justify your personal beliefs. When you combine that with a blatant disregard for the fundamental teaching of the faith tradition, you end up with a perverse, dangerous distortion which can fuel hatred and violence in direct contradiction to the teaching of the faith".

Muslims, a religiously, culturally, nationally and socioeconomically diverse faith-based populace, bear no exception in escaping this dilemma[7-9]. Many abusers use religion and culture as an excuse to justify their actions despite obvious evidence opposing this behavior.

Yet, like in many faith communities, it is only in the recent past receiving attention as a major concern in North America and globally. Some scholars suggest that the global Muslim community is in the early stages of considering IPV as a social problem of criminal nature [10-
12]. This is also evident in the fact that no empirical articles written in English were found on this issue prior to the 1990s. Like many faith communities, the subject of IPV in Muslims communities generally tends to remain a taboo; there is either general reluctance or denial that IPV exists $[10,13,14]$. It may remain a topic hidden within many families and, once again, this guarded nature is reflected in the limited availability of research on this issue within the professional literature $[8,15]$. However, the works of a few activists, academics and grassroots level agencies have helped shed light on the gravity of this global issue in more recent times [8,15-21]. These advocates, as part of an emerging movement, are revealing that IPV is a major problem afflicting Muslim families similar to any other faith community $[15,22,23]$. They have also highlighted that Muslims, as a faith, culture, and sociopolitical community, have special and additional intersecting factors that complicate the contextual circumstances of IPV that involve their varied religious and cultural traditions $[15,24]$.

Much can be learned from empirical studies on this subject, as these are essential for social scientists to understand a phenomenon scientifically. Not only is there a paucity of literature on the topic, but no critical synthesis of empirical studies has been done on this topic to more fully understand the empirical strengths, limitations and gaps in the literature. The goal of a systematic review is to attempt to organize and evaluate study findings using pre-determined procedures. This reduces the chance for bias and allows for future replication. The studies used are evaluated for quality, allowing their findings to be more reliably combined.This process allows the researcher to provide more reliable findings that can be used to guide their recommendations and

*Corresponding author: Randall Nedegaard, University of North Dakota, Grand Forks, ND, USA, Tel: 701-777-3766; E-mail: randall.nedegaard@und.edu

Received February 03, 2014; Accepted June 26, 2014; Published July 03, 2014

Citation: Jayasundara D, Nedegaard R, Sharma B, Flanagan K (2014) Intimate Partner Violence in Muslim Communities. Arts Social Sci J S1: 003. doi: 10.4172/2151-6200.S1-003

Copyright: @ 2014 Jayasundara D, et al. This is an open-access article distributed under the terms of the Creative Commons Attribution License, which permits unrestricted use, distribution, and reproduction in any medium, provided the original author and source are credited. 
conclusions (Cochrane, n.d.; Campbell Corporation, n.d.). As a first step towards remedying this deficit, this paperconducts an extensive, systematic literature review on current material regarding Muslims and IPV in order to illuminate more fully the complexities, intersections and unique features involved. While this study was mainly written for social scientists in North America who are interested in partner abuse in Muslim communities, the literature reviewed in this study included both North American and global empirical studies to help provide a more comprehensive understanding of the issue. This paper will provide insight as to what areas were studied, what gaps exist in literature, and implications for future studies.

The primary research question addressed in this study asks what do past empirical studies say about IPV among Muslims? To understand this question, this study develops an overview of the findings of the past studies that empirically examined the topic of IPV among Muslims globally.

\section{Methods}

This review meets the PRISMA [25] guidelines for systematic reviews in its assessments of topic areas covered, critique of methodologies used, documentation of results and the identification of gaps for future research. The study authors met repeatedly to develop the research question, inclusion and exclusion criteria, and to identify search terms in order to conduct this review. To prevent bias, prior to starting the project, the authors identified the definition and scope for the study and created an initial table that included the inclusion and exclusion criteria listed below (table available upon request).

\section{Inclusion and Exclusion Criteria}

The following inclusion criteria are utilized to narrow down the study articles: 1) for scope of the current study, IPV is defined as all forms of violence between married and/or adult intimate partners. Therefore this study is limited to IPV among spouses and only includes related extended family violence when both their partner and other family members abuse the victim, or when the definition of partner abuse in a study includes abuse by extended family members. Consequently, studies that looked at any form of abuse by partners are included. Empirical studies are also included if they 2) meet at least one of the following three criteria: (a) they looked solely at IPV in the Muslim population and/or; (b) they are comparative studies that control for religion as a primary purpose of the study, and discuss in detail about study implications to Muslim communities, and/or; (c) at least $75 \%$ of the study participants or more are identified as Muslims to be able to generalize to Muslim communities. As mentioned previously, 3) no restrictions are placed on the country of the study, hence, all globally available published research reports in English are included and; 4) all reports that discuss their methodology are considered.For example, two online prevalence surveys and one technical report are included. Non-refereed empirical articles are included due to the scarcity of refereed articles in the preliminary literature search on the topic of IPV and to gain a more comprehensive understanding on the topic in general by including gray literature such as dissertations and other relevant technical reports.Finally, 5) no date restrictions are imposed given the paucity of studies. The earliest study found to meet these criteria is in 1998.

As for specific exclusion criteria, 1) studies primarily on extended family abuse without a main research focus on intimate partner abuse are excluded (for example, some honor killing studies that did not focus on IPV are not included); 2) studies that did not specifically mention if their participants are Muslim, even if they are conducted in primarily Muslim countries (many studies conducted in middle eastern countries separated their data by religion, therefore, the authors made a decision to leave out articles that do not mention religion as the subject or participant's religion). Finally, 3) one dissertation is excluded because the author denied access.

\section{Sources and Methods of Data Extraction}

Data collection utilized the following electronic databases: Academic Search Premier, Anthropology Plus, Applied Social Science Index and Abstracts, Books in Print, CAB Abstracts, Campbell Library Choice, Cochrane Library, Criminal Justice Abstracts, Directory of Open Access Journals, Ebsco Research data bases, Expanded Academic ASAP, Google Scholar, Health Source, Highwire Press, InformaWorld, International Bibliography of Social Science, Medline, Proquest, Psych Info, Sage Journals online, Social Services, Social Work, and Sociology Abstracts. Additionally, the Asian \& Pacific Islander Institute for DV, the Faith Trust Institute, and the Institute for Social Policy and Understanding web resources were also investigated due to their extensive work with Muslim communities. Search terms used were "Domestic Violence and Muslims/Islam", "Muslims/Islam and Spousal Abuse", "Intimate Partner Violence and Muslims/Islam", and "Interpersonal Violence and Muslims/Islam".

The articles were gathered by two graduate research assistants and followed-up by the authors for triangulation purposes. Initial searches yielded multiple hits over 200. Screening out took place in several stages.At first, screening involved gathering all available documents that discussed IPV among Muslim communities. This process identified over 80 articles, technical reports and books. After gathering the documents, two of the authors separately looked at each of the articles to ensure they met the above-mentioned inclusion and exclusion criteria. After careful screening by the authors, only 35 articles met the criteria originally set forth (Figure 1).

\section{Assessment for Quality}

The study critically appraises and synthesizes existing literature on the topic. As the studies identified were non-experimental, qualitative, or mix-methods in nature and the majority of the scales are assessments of quantitative articles only, the authors made a decision to create their own scale of assessment guided by Zazaet al. [26] Guide to Community Preventative Services and Greeret al. guide for Grading [27]. Reynoldset al. [28] recommends against use of a pre-identified quality assurance measures for qualitative studies, hence this study uses guidelines standard for measuring the rigor of qualitative methods. All authors separately rated the scales and, if discrepancies were identified, they were resolved through discussion and reanalysis. For the purposes of rating categories, the following criteria are used.

The first criteria assessed the question: was the study design clearly outlined? The authors reviewed the articles to identify if the following areas are clearly addressed in the papers: research questions, sampling methods, units of analysis, sample size, validity and reliability discussions, analysis of methods discussed, and finally the appropriateness of the design employed based on the research questions.This method was used for quantitative, qualitative, and mix method designs. The ratings are based on whether or not the studies met all the criteria or some of the criteria. Quantitative studies demonstrating significant weaknesses are ranked as weak. For qualitative studies the papers are assessed based on their description 


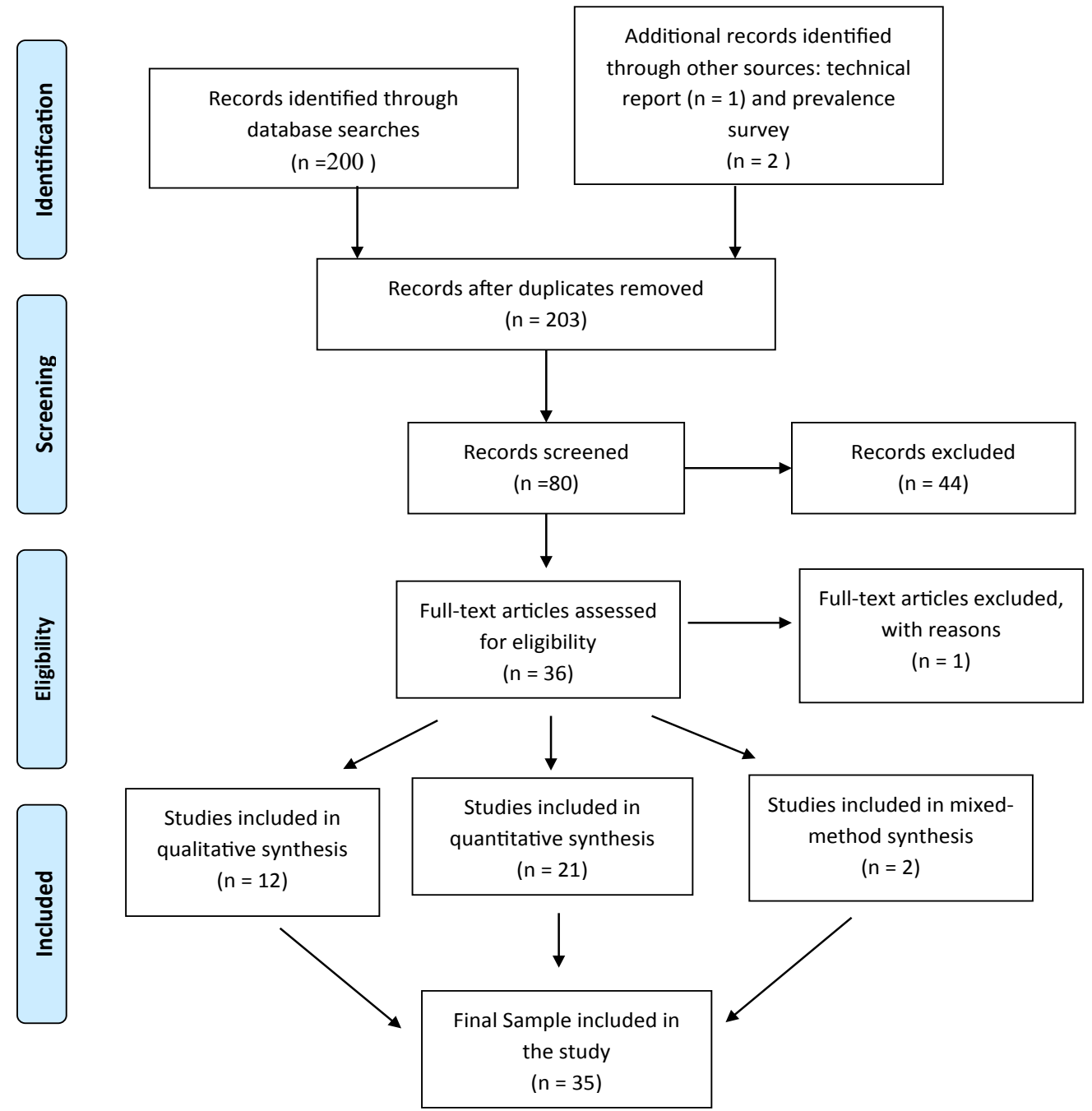

Figure 1: Flow diagram of studies for systematic review

of methods (accuracy; recording and transcribing), interpretation of results (using bracketing, open-ended questions, journaling methods), and for theory (alternate explanations). Once again, the ratings are based on whether the studies met all the criteria or some of the criteria. Qualitative studies demonstrating significant weaknesses are also ranked as weak.

The second criteria assessed for measurement error. For quantitative studies, the measurement error is ranked based on the validity and reliability reported for the measures used when applicable. For validity, the study looks at whether a validity scale is used and, when necessary, if revalidation using exploratory or confirmatory factor analysis methods were applied. Similarly for the reliability, the articles are scanned to determine if reliability scales were used and, when applicable, if they tested/or retested using reliability tests such as Chronbach's Alpha. The rankings are based on whether both validity and reliability criteria are met, some are met, or none are met or discussed. Qualitative studies are graded for validity based on whether or not they used the following types of validity testing methods: long term participant observation, feedback from clients, member checks, peer review, negative case analysis, and whether theoretical sampling methods are used. Reliability is graded based upon the use of a second coder or if any software was utilized. This ranking is based on whether validity and reliability criteria are both met, some are met, or none are met or discussed.

The third criteria looked at: appropriateness of the analysis. All articles are assessed for appropriateness of analysis based upon whether the methods of analysis used are appropriate to answer the research question/s and based on the research design employed. This ranking is based on the superior level of analysis methods used to meet research questions based on the design utilized.

Fourthly, interpretation of results are assessed, the interpretation of results for quantitative studies is based on whether or not the studies took into consideration all controllable confounding variables in their design and discussed the study limitations for factors they were unable to control for. This ranking is based on the extent of interpretation. For qualitative methods, the interpretation of results is ranked based on the coherence and communicability of the analysis of those results.

Finally, the paper assessed for generalizability/transferability of studies. In quantitative studies the ranking was based on the extent of generalizability. Quantitative studies using random sampling methods 
Citation: Jayasundara D, Nedegaard R, Sharma B, Flanagan K (2014) Intimate Partner Violence in Muslim Communities. Arts Social Sci J S1: 003. doi: 10.4172/2151-6200.S1-003

are considered to have high generalizability. Studies employing nonrandom sampling methods are considered to have low generalizability. If higher rigor non-random sampling methods are used, such as quarter sampling, the generalizability is considered moderate. For studies using secondary data, this score is based on the sampling method used in the surveys they utilized.For qualitative studies, the concept of transferability of content, such as abstract constructs, is used as a guiding principal for ranking of data.

\begin{tabular}{|c|c|c|c|c|c|c|c|}
\hline Author/s & Title \& Year & Study Site & $\begin{array}{l}\text { Main Themes } \\
\text { addressed } \\
\text { related to the } \\
\text { review }\end{array}$ & $\begin{array}{l}\text { Unit of } \\
\text { analysis \& } \\
\text { sample size } \\
\text { or power } \\
\text { calculations }\end{array}$ & \begin{tabular}{l|} 
Research \\
Design, Data \\
Source and \\
Sampling \\
Method
\end{tabular} & $\begin{array}{l}\text { Measures, Validity and } \\
\text { Reliability }\end{array}$ & Strengths and limitations \\
\hline \multicolumn{8}{|l|}{ Qualitative } \\
\hline $\begin{array}{l}\text { Zakar, R., } \\
\text { Zakar, M. } \\
\text { Z., Faist, T. } \\
\text { \& Kraemer, } \\
\text { A. }\end{array}$ & $\begin{array}{l}\text { Intimate partner violence } \\
\text { against women and } \\
\text { its related immigration } \\
\text { stressors in Pakistani } \\
\text { immigrant families in } \\
\text { Germany, } 2012\end{array}$ & Germany & $\begin{array}{l}\text { Factors } \\
\text { associated with } \\
\text { DV; Attitudes } \\
\text { Towards DV; } \\
\text { Experiences } \\
\text { of Coping and } \\
\text { Support }\end{array}$ & $\begin{array}{l}\text { Individual, } \\
32 \text { Immigrant } \\
\text { Pakistani } \\
\text { women, } 96.8 \% \\
\text { were Muslim }\end{array}$ & $\begin{array}{l}\text { Qualitative, } \\
\text { Primary, } \\
\text { Snowball } \\
\text { sampling }\end{array}$ & $\begin{array}{l}\text { Semi-structured interview } \\
\text { guides } \\
\text { Tape recorded interviews, } \\
\text { multiple authors coding, } \\
\text { and member checks }\end{array}$ & $\begin{array}{l}\text { Meaningfully contributes to } \\
\text { understanding of IPV in the } \\
\text { context of immigration; Validity } \\
\text { and reliability discussed; } \\
\text { Using mixed methods data could } \\
\text { improve the study; Transferability } \\
\text { and synthesis of interpretations } \\
\text { can be improved }\end{array}$ \\
\hline $\begin{array}{l}\text { Desai, S. \& } \\
\text { Haffajee, Z. }\end{array}$ & $\begin{array}{l}\text { Breaking the silence: } \\
\text { reclaiming Qur'anic } \\
\text { interpretations as a tool } \\
\text { for empowerment and } \\
\text { liberatory praxis for } \\
\text { dealing with domestic } \\
\text { violence in Canadian } \\
\text { Muslim communities, } \\
2011\end{array}$ & Canada & $\begin{array}{l}\text { Factors } \\
\text { associated with } \\
\text { DV; Experiences } \\
\text { with coping and } \\
\text { support }\end{array}$ & $\begin{array}{l}\text { Individual, } 2 \\
\text { case studies, } \\
\text { both were } \\
\text { Muslim Women }\end{array}$ & $\begin{array}{l}\text { Qualitative, } \\
\text { Primary, } \\
\text { Convenience } \\
\text { sampling }\end{array}$ & $\begin{array}{l}\text { In-depth interviews } \\
\text { No discussion of validity } \\
\text { and reliability }\end{array}$ & $\begin{array}{l}\text { Weak case study design method; } \\
\text { limited transferability } \\
\text { Strength: Deeper understanding } \\
\text { through case study design the } \\
\text { experiences of two women and } \\
\text { their theoretical implications }\end{array}$ \\
\hline $\begin{array}{l}\text { Kulwicki, A., } \\
\text { Hymes, R., } \\
\text { Hammad, } \\
\text { A., Killawi, } \\
\text { A., \& } \\
\text { Farrag, M. }\end{array}$ & $\begin{array}{l}\text { Development of a } \\
\text { Domestic Violence Risk } \\
\text { Assessment Tool for } \\
\text { Arab American Clients, } \\
2010\end{array}$ & $\begin{array}{l}\text { United } \\
\text { States }\end{array}$ & $\begin{array}{l}\text { Scale } \\
\text { development }\end{array}$ & $\begin{array}{l}\text { Focus group } \\
\text { level, } 5 \text { focus } \\
\text { groups Focus } \\
\text { groups specify } \\
\text { and differentiate } \\
\text { religious issues } \\
\text { among Arab } \\
\text { Descents }\end{array}$ & $\begin{array}{l}\text { Qualitative, } \\
\text { Primary, } \\
\text { Purposeful } \\
\text { sampling }\end{array}$ & $\begin{array}{l}\text { Interview guides developed } \\
\text { by the authors } \\
\text { Interviews were audiotaped } \\
\text { and transcribed } \\
\text { Both manual and computer } \\
\text { analysis were conducted } \\
\text { Two coders and member } \\
\text { checks were used } \\
\text { Kreugers focus group } \\
\text { criteria used }\end{array}$ & $\begin{array}{l}\text { Study identified important DV } \\
\text { concepts, however, the study } \\
\text { did not lead to completion of a } \\
\text { standardized risk assessment }\end{array}$ \\
\hline $\begin{array}{l}\text { GharaibeH, } \\
\text { M., \& } \\
\text { Oweis, A. }\end{array}$ & $\begin{array}{l}\text { Why Jordanian women } \\
\text { stay in an abusive } \\
\text { relationship: Implications } \\
\text { to health and well-being, } \\
2009\end{array}$ & Jordan & $\begin{array}{l}\text { Experiences } \\
\text { of Coping and } \\
\text { Support }\end{array}$ & $\begin{array}{l}\text { Individual, } \\
28 \text { Jordanian } \\
\text { women } \\
\text { who have } \\
\text { experienced } \\
\text { violence. All } \\
\text { were Muslim. }\end{array}$ & $\begin{array}{l}\text { Qualitative, } \\
\text { Primary, } \\
\text { Purposeful } \\
\text { snow-ball } \\
\text { sampling }\end{array}$ & $\begin{array}{l}\text { Open-ended face to face } \\
\text { interview } \\
\text { In-depth interviews, thick } \\
\text { description, tape-recorded } \\
\text { and transcribed. }\end{array}$ & $\begin{array}{l}\text { Addresses an important topic with } \\
\text { implications to human service } \\
\text { providers } \\
\text { Study can be followed up with } \\
\text { mixed methods to strengthen } \\
\text { further transferability }\end{array}$ \\
\hline Snajdr, E. & $\begin{array}{l}\text { Gender, power, and the } \\
\text { performance } \\
\text { of justice: } \\
\text { Muslim women's } \\
\text { responses to domestic } \\
\text { violence } \\
\text { in Kazakhstan, } \\
2005\end{array}$ & Kazakhstan & Service Response & $\begin{array}{l}\text { Individual, Field } \\
\text { observations of } \\
\text { Muslim women, } \\
\text { ethnographic } \\
\text { method, number } \\
\text { not mentioned }\end{array}$ & $\begin{array}{l}\text { Qualitative, } \\
\text { Primary, Field } \\
\text { observations }\end{array}$ & $\begin{array}{l}\text { Field observations } \\
\text { No discussion of validity } \\
\text { and reliability }\end{array}$ & $\begin{array}{l}\text { Study conducted in Kazakhstan } \\
\text { using ethnographic methods, } \\
\text { no discussion of validity and } \\
\text { reliability the field method used }\end{array}$ \\
\hline $\begin{array}{l}\text { Hassouneh- } \\
\text { Phillips, D. }\end{array}$ & $\begin{array}{l}\text { Strength and vulnerability: } \\
\text { Spirituality in abused } \\
\text { American Muslim } \\
\text { women's lives, } 2003\end{array}$ & $\begin{array}{l}\text { United } \\
\text { States }\end{array}$ & $\begin{array}{l}\text { Experiences } \\
\text { of Coping and } \\
\text { Support }\end{array}$ & $\begin{array}{l}\text { Individual, } \\
17 \text { abused } \\
\text { American } \\
\text { Muslim women }\end{array}$ & $\begin{array}{l}\text { Qualitative, } \\
\text { Primary, } \\
\text { Purposeful } \\
\text { sampling }\end{array}$ & $\begin{array}{l}\text { Interviews. Member } \\
\text { checks, prolonged } \\
\text { engagement, peer review, } \\
\text { external audit, triangulation, } \\
\text { and rich thick description }\end{array}$ & $\begin{array}{l}\text { Important topic. Future studies } \\
\text { can replicate this study using mix } \\
\text { methods }\end{array}$ \\
\hline $\begin{array}{l}\text { Hassouneh- } \\
\text { Phillips, D. }\end{array}$ & $\begin{array}{l}\text { American Muslim } \\
\text { Women's Experiences } \\
\text { of leaving abusive } \\
\text { relationships, 2001a }\end{array}$ & $\begin{array}{l}\text { United } \\
\text { States }\end{array}$ & $\begin{array}{l}\text { Prevalence and } \\
\text { Types of Abuse }\end{array}$ & $\begin{array}{l}\text { Individual, } \\
17 \text { American } \\
\text { Muslim women } \\
\text { who have left } \\
\text { their partners }\end{array}$ & $\begin{array}{l}\text { Qualitative, } \\
\text { Primary, } \\
\text { Purposeful } \\
\text { sampling }\end{array}$ & $\begin{array}{l}\text { Focus groups and } \\
\text { individual interview using } \\
\text { unstructured interview } \\
\text { guides } \\
\text { Use of member } \\
\text { checks, prolonged } \\
\text { engagement, peer review, } \\
\text { external audit, triangulation, } \\
\text { and rich thick description }\end{array}$ & $\begin{array}{l}\text { Extremely important topic, } \\
\text { but how this process impacts } \\
\text { services utilization is not } \\
\text { discussed in detail }\end{array}$ \\
\hline $\begin{array}{l}\text { Hassouneh- } \\
\text { Phillips, } \\
\text { D. S }\end{array}$ & $\begin{array}{l}\text { Marriage is half faith } \\
\text { and rest is fear of Allah: } \\
\text { marriage and spouse } \\
\text { abuse among American } \\
\text { Muslims, 2001b }\end{array}$ & $\begin{array}{l}\text { United } \\
\text { States }\end{array}$ & $\begin{array}{l}\text { Experiences } \\
\text { of Coping and } \\
\text { Support }\end{array}$ & $\begin{array}{l}\text { Individual, } \\
\text { interviews from } \\
17 \text { American } \\
\text { Muslim women, } \\
\text { interpretive } \\
\text { phenomenology }\end{array}$ & $\begin{array}{l}\text { Primary, } \\
\text { Qualitative, } \\
\text { Purposeful } \\
\text { sampling }\end{array}$ & $\begin{array}{l}\text { Unstructured, group and } \\
\text { individual interviews } \\
\text { Follow-up interviews, } \\
\text { external auditor, member } \\
\text { checks, thick description, } \\
\text { peer review }\end{array}$ & $\begin{array}{l}\text { Raises awareness on cultural } \\
\text { differences on marriage and DV } \\
\text { future studies can replicate this } \\
\text { study }\end{array}$ \\
\hline
\end{tabular}


Citation: Jayasundara D, Nedegaard R, Sharma B, Flanagan K (2014) Intimate Partner Violence in Muslim Communities. Arts Social Sci J S1: 003. doi: 10.4172/2151-6200.S1-003

Page 5 of 12

\begin{tabular}{|c|c|c|c|c|c|c|c|}
\hline $\begin{array}{l}\text { Hassouneh- } \\
\text { Phillips, D. }\end{array}$ & $\begin{array}{l}\text { Polygamy and wife } \\
\text { abuse: A qualitative study } \\
\text { of Muslim women in } \\
\text { America, 2001c }\end{array}$ & $\begin{array}{l}\text { United } \\
\text { States }\end{array}$ & $\begin{array}{l}\text { Experiences } \\
\text { of Coping and } \\
\text { Support }\end{array}$ & $\begin{array}{l}\text { Individual, } \\
17 \text { American } \\
\text { Muslim women }\end{array}$ & $\begin{array}{l}\text { Qualitative, } \\
\text { Primary, } \\
\text { Purposeful } \\
\text { sampling }\end{array}$ & $\begin{array}{l}\text { Interview. Member } \\
\text { checks, prolonged } \\
\text { engagement, peer review, } \\
\text { external audit, triangulation, } \\
\text { and rich thick description }\end{array}$ & $\begin{array}{l}\text { Important topic } \\
\text { Future studies can replicate this } \\
\text { study using mix methods }\end{array}$ \\
\hline $\begin{array}{l}\text { Ammar, } \\
\text { N. H. }\end{array}$ & $\begin{array}{l}\text { Simplistic Stereotyping } \\
\text { and Complex Reality of } \\
\text { Arab-American Immigrant } \\
\text { Identity: consequences } \\
\text { and future strategies in } \\
\text { policing wife battery, } 2000\end{array}$ & $\begin{array}{l}\text { United } \\
\text { States }\end{array}$ & Service Response & $\begin{array}{l}\text { Individual, } 4 \\
\text { case study } \\
\text { interviews with } \\
\text { police officers }\end{array}$ & $\begin{array}{l}\text { Qualitative, } \\
\text { Primary, } \\
\text { Convenience } \\
\text { sampling }\end{array}$ & $\begin{array}{l}\text { Police records and } \\
\text { interviews } \\
\text { Triangulation, thick } \\
\text { description }\end{array}$ & $\begin{array}{l}\text { Addressed a topic rarely looked } \\
\text { at, case study approach was } \\
\text { appropriate for the topic } \\
\text { Transferability limitations }\end{array}$ \\
\hline $\begin{array}{l}\text { Keenan, } \\
\text { C. K., El- } \\
\text { Hadad, A. } \\
\text { \& Balian, } \\
\text { S. A. }\end{array}$ & $\begin{array}{l}\text { Factors Associated with } \\
\text { Domestic Violence in } \\
\text { Low-Income Lebanese } \\
\text { Families, } 1998 .\end{array}$ & Lebanon & $\begin{array}{l}\text { Prevalence } \\
\text { and Type of } \\
\text { Abuse; Factors } \\
\text { Associated with } \\
\text { DV }\end{array}$ & $\begin{array}{l}\text { Individual, } \\
60 \text { women in } \\
\text { low-income } \\
\text { Christian and } \\
\text { Muslim families } \\
\text { Study controls } \\
\text { for religion }\end{array}$ & $\begin{array}{l}\text { Qualitative, } \\
\text { Primary, } \\
\text { Purposeful } \\
\text { sampling }\end{array}$ & $\begin{array}{l}\text { Semi-structured interviews } \\
\text { Long interviews were tape- } \\
\text { recorded } \\
\text { Use of software } \\
\text { Validity checks by } \\
\text { consultants for credibility, } \\
\text { authenticity and plausibility }\end{array}$ & $\begin{array}{l}\text { Important topic } \\
\text { Extensive checks for validity and } \\
\text { reliability } \\
\text { Use of mixed methods can be } \\
\text { used for future studies related to } \\
\text { this topic; stronger synthesis can } \\
\text { strengthen the study }\end{array}$ \\
\hline \multicolumn{8}{|c|}{ Mixed Methods } \\
\hline $\begin{array}{l}\text { Btoush, R. \& } \\
\text { Haj-Yahia, } \\
\text { M. M. }\end{array}$ & $\begin{array}{l}\text { Attitudes of Jordanian } \\
\text { Society Toward Wife } \\
\text { Abuse, } 2008 .\end{array}$ & Jordan & $\begin{array}{l}\text { Attitudes Towards } \\
\text { DV; Prevalence; } \\
\text { Experiences } \\
\text { of Coping and } \\
\text { Support }\end{array}$ & $\begin{array}{l}\text { Individual, } 260 \\
\text { Jordanian men } \\
\text { and women. } \\
97 \% \text { were } \\
\text { Muslim }\end{array}$ & $\begin{array}{l}\text { Mixed } \\
\text { Methods, } \\
\text { Primary, } \\
\text { Convenience } \\
\text { sampling }\end{array}$ & $\begin{array}{l}\text { Self-administered open } \\
\text { and closed ended } \\
\text { questionnaires } \\
\text { No discussion of validity } \\
\text { and reliability }\end{array}$ & $\begin{array}{l}\text { Mix method design increases } \\
\text { understanding, however, } \\
\text { non-random sampling limits } \\
\text { generalizability, no discussion of } \\
\text { validity and reliability }\end{array}$ \\
\hline $\begin{array}{l}\text { Haj-Yahia, } \\
\text { M. }\end{array}$ & $\begin{array}{l}\text { Wife abuse and battering } \\
\text { in the sociocultural } \\
\text { context of Arab society, } \\
2000\end{array}$ & Israel & $\begin{array}{l}\text { Attitudes Towards } \\
\text { DV }\end{array}$ & $\begin{array}{l}\text { Individual, } 291 \\
\text { women from } \\
\text { one study } 2,102 \\
\text { married Arab } \\
\text { Israeli women } \\
\text { (75\% Muslim) }\end{array}$ & $\begin{array}{l}\text { Mixed } \\
\text { Methods, } \\
\text { Primary, } \\
\text { Convenience } \\
\text { sampling } \\
\text { and random } \\
\text { sampling }\end{array}$ & $\begin{array}{l}\text { Using two surveys with two } \\
\text { different populations } \\
\text { No discussion of validity } \\
\text { and reliability }\end{array}$ & $\begin{array}{l}\text { Using mix methods, validity and } \\
\text { reliability is not discussed }\end{array}$ \\
\hline \multicolumn{8}{|l|}{ Quantitative } \\
\hline $\begin{array}{l}\text { Dalal, K., } \\
\text { Andrews, J. } \\
\text { \& Dawad, S. }\end{array}$ & $\begin{array}{l}\text { Contraception Use and } \\
\text { Associations with Intimate } \\
\text { Partner Violence Among } \\
\text { Women in Bangladesh, } \\
2012\end{array}$ & Bangladesh & $\begin{array}{l}\text { DV and Health } \\
\text { Consequences; } \\
\text { Prevalence }\end{array}$ & $\begin{array}{l}\text { Individual, } \\
10996 \text { ( } 9924 \\
\text { Muslim) } \\
\text { women living } \\
\text { in Bangladesh. } \\
\text { Study controls } \\
\text { for religion }\end{array}$ & $\begin{array}{l}\text { Quantitative, } \\
\text { Secondary }\end{array}$ & $\begin{array}{l}\text { Bangladesh democratic } \\
\text { health survey } \\
\text { No discussion of validity } \\
\text { and reliability }\end{array}$ & $\begin{array}{l}\text { Use of a national representative } \\
\text { survey increases generalizability, } \\
\text { however validity and reliability } \\
\text { was not discussed }\end{array}$ \\
\hline $\begin{array}{l}\text { Haj-Yahia, } \\
\text { M. M., } \\
\text { Wilson, R. } \\
\text { M. \& Naqvi, } \\
\text { S. A. M. }\end{array}$ & $\begin{array}{l}\text { Justification, Perception } \\
\text { of Severity and Harm, and } \\
\text { Criminalization of Wife } \\
\text { Abuse in the Palestinian } \\
\text { Society, } 2012\end{array}$ & $\begin{array}{l}\text { West Bank } \\
\text { and Gaza } \\
\text { Strips }\end{array}$ & $\begin{array}{l}\text { Attitudes Towards } \\
\text { DV }\end{array}$ & $\begin{array}{l}\text { Individual, } \\
624 \text { adult } \\
\text { Palestinian men } \\
\text { and women } \\
\text { from the West } \\
\text { Bank and Gaza } \\
\text { Strip. } 93 \% \text { of } \\
\text { the sample } \\
\text { were Muslim }\end{array}$ & $\begin{array}{l}\text { Quantitative, } \\
\text { Primary, } \\
\text { Systematic } \\
\text { random } \\
\text { sampling }\end{array}$ & $\begin{array}{l}\text { Adopted existing } \\
\text { instruments for use in the } \\
\text { study } \\
\text { Reliability tested, validity } \\
\text { not discussed }\end{array}$ & $\begin{array}{l}\text { Use of random sampling } \\
\text { increases generalizability } \\
\text { Adopted existing scales to } \\
\text { enhance cultural competence } \\
\text { Discusses validity and reliability. }\end{array}$ \\
\hline $\begin{array}{l}\text { Rahman, } \\
\text { M., } \\
\text { Sasagawa, } \\
\text { T., Fujii, R, } \\
\text { Tomizawa, } \\
\text { H. \& } \\
\text { Makinoda, } \\
\text { S. }\end{array}$ & $\begin{array}{l}\text { Intimate Partner } \\
\text { Violence and Unintended } \\
\text { Pregnancy Among } \\
\text { Bangladeshi Women, } \\
2012\end{array}$ & Bangladesh & $\begin{array}{l}\text { Prevalence; } \\
\text { DV and Health } \\
\text { Consequences }\end{array}$ & $\begin{array}{l}\text { Individual, } \\
4695 \text { married } \\
\text { Bangladeshi } \\
\text { women. Study } \\
\text { controls for } \\
\text { religion when } \\
\text { reporting IPV } \\
\text { percentages }\end{array}$ & $\begin{array}{l}\text { Quantitative, } \\
\text { Secondary }\end{array}$ & $\begin{array}{l}\text { National Bangladesh } \\
\text { democratic health survey } \\
\text { No discussion of validity } \\
\text { and reliability }\end{array}$ & $\begin{array}{l}\text { Use of national representative } \\
\text { survey increases generalizability } \\
\text { No discussion of validity and } \\
\text { reliability }\end{array}$ \\
\hline $\begin{array}{l}\text { Celik, A. \& } \\
\text { Sabri, B. }\end{array}$ & $\begin{array}{l}801 \text { Muslims Speak Up } \\
\text { to Stop Family Violence, } \\
2011\end{array}$ & Unite States & $\begin{array}{l}\text { Prevalence; } \\
\text { Attitudes Towards } \\
\text { DV }\end{array}$ & $\begin{array}{l}\text { Individual, } 801 \\
\text { Muslim men } \\
\text { and women }\end{array}$ & $\begin{array}{l}\text { Quantitative, } \\
\text { Primary, } \\
\text { Purposeful } \\
\text { sampling }\end{array}$ & $\begin{array}{l}\text { Online survey developed } \\
\text { by authors } \\
\text { No discussion of validity } \\
\text { and reliability }\end{array}$ & $\begin{array}{l}\text { Covers an important area } \\
\text { Non-random sampling limits } \\
\text { generalizability, details of design } \\
\text { and methodology were not } \\
\text { discussed as it was presented in } \\
\text { a report form }\end{array}$ \\
\hline $\begin{array}{l}\text { Haddad, } \\
\text { L. G., } \\
\text { Shotar, A., } \\
\text { Younger, J. } \\
\text { B., Alzyoud, } \\
\text { S. \& } \\
\text { Bouhaidar, } \\
\text { C. M. }\end{array}$ & $\begin{array}{l}\text { Screening for domestic } \\
\text { violence in Jordan: } \\
\text { validation of an Arabic } \\
\text { version of a domestic } \\
\text { violence against women } \\
\text { questionnaire, } 2011 .\end{array}$ & Jordan & $\begin{array}{l}\text { Prevalence; Scale } \\
\text { Validation }\end{array}$ & $\begin{array}{l}\text { Individual, } \\
175 \text { Jordanian } \\
\text { women, } 90 \% \\
\text { were Muslim }\end{array}$ & $\begin{array}{l}\text { Quantitative, } \\
\text { Primary, } \\
\text { Convenience } \\
\text { sampling }\end{array}$ & $\begin{array}{l}\text { NorVold Domestic Abuse } \\
\text { Questionnaire (NORAQ) - } \\
\text { Arabic version } \\
\text { Tested reliability and } \\
\text { validity as the primary focus }\end{array}$ & $\begin{array}{l}\text { Validated an important tool that } \\
\text { will have application beyond } \\
\text { Jordan to other Middle Eastern } \\
\text { countries. Further research } \\
\text { could follow-up with qualitative } \\
\text { experiences with the instrument } \\
\text { capturing the women's reality } \\
\text { Non-random sampling limits } \\
\text { generalizability }\end{array}$ \\
\hline
\end{tabular}


Citation: Jayasundara D, Nedegaard R, Sharma B, Flanagan K (2014) Intimate Partner Violence in Muslim Communities. Arts Social Sci J S1: 003. doi: 10.4172/2151-6200.S1-003

\begin{tabular}{|c|c|c|c|c|c|c|c|}
\hline $\begin{array}{l}\text { Yount, K.M. } \\
\text { \& Li, L. }\end{array}$ & $\begin{array}{l}\text { Domestic Violence } \\
\text { Against Married Women } \\
\text { in Egypt, } 2010\end{array}$ & Egypt & $\begin{array}{l}\text { Prevalence; } \\
\text { Factors } \\
\text { Associated with } \\
\text { DV }\end{array}$ & $\begin{array}{l}\text { Individual, } \\
5272 \text { married } \\
\text { Egyptian } \\
\text { Women. Study } \\
\text { controls for } \\
\text { religion for } \\
\text { prevalence } \\
\text { rates }\end{array}$ & $\begin{array}{l}\text { Quantitative, } \\
\text { Secondary }\end{array}$ & $\begin{array}{l}\text { DHS National Survey. No } \\
\text { discussion of validity and } \\
\text { reliability }\end{array}$ & $\begin{array}{l}\text { Marital resources and constraints } \\
\text { accounted for most of the risk for } \\
\text { physical DV } \\
\text { No differences were identified } \\
\text { based on religion. National } \\
\text { representative sample } \\
\text { Validity and reliability not } \\
\text { discussed }\end{array}$ \\
\hline $\begin{array}{l}\text { Dalal, K., } \\
\text { Rahman,F. } \\
\text { \& Jansson, } \\
\text { B. }\end{array}$ & $\begin{array}{l}\text { Wife Abuse in Rural } \\
\text { Bangladesh, } 2009\end{array}$ & Bangladesh & $\begin{array}{l}\text { Prevalence; } \\
\text { Factors } \\
\text { Associated with } \\
\text { DV }\end{array}$ & $\begin{array}{l}\text { Individual, } 4411 \\
\text { married rural } \\
\text { Bangladeshi } \\
\text { women. Study } \\
\text { controls for } \\
\text { religion for } \\
\text { prevalence } \\
\text { rates }\end{array}$ & $\begin{array}{l}\text { Quantitative, } \\
\text { Primary, } \\
\text { Random } \\
\text { selection }\end{array}$ & $\begin{array}{l}\text { Pretested questionnaires } \\
\text { created for the study } \\
\text { design Only mentions the } \\
\text { measures were pretested } \\
\text { No other discussion of } \\
\text { reliability or validity }\end{array}$ & $\begin{array}{l}\text { Random representative sample } \\
\text { increases generalizability } \\
\text { No discussion of validity or } \\
\text { reliability of measures used }\end{array}$ \\
\hline Ghayyur, T & $\begin{array}{l}\text { Domestic Violence Survey } \\
\text { Analysis February-March, } \\
2009\end{array}$ & Unite States & Prevalence & $\begin{array}{l}\text { Individual, } 241 \\
\text { Muslim men } \\
\text { and women } \\
\text { living in the US }\end{array}$ & $\begin{array}{l}\text { Quantitative, } \\
\text { Primary, } \\
\text { Non-random } \\
\text { sampling }\end{array}$ & $\begin{array}{l}\text { Online survey developed } \\
\text { for the study } \\
\text { No discussion of validity } \\
\text { and reliability }\end{array}$ & $\begin{array}{l}\text { Covers an important area Non- } \\
\text { random sampling, details of } \\
\text { design and methodology was not } \\
\text { discussed as it was presented in } \\
\text { a report form }\end{array}$ \\
\hline $\begin{array}{l}\text { Okenwa, L. } \\
\text { E., Lawoko, } \\
\text { S. \& } \\
\text { Jansson, B. }\end{array}$ & $\begin{array}{l}\text { Exposure to Intimate } \\
\text { Partner Violence Amongst } \\
\text { Women of Reproductive } \\
\text { Age in Lagos, Nigeria: } \\
\text { Prevalence and } \\
\text { Predictors, 2009. }\end{array}$ & Nigeria & $\begin{array}{l}\text { Prevalence; } \\
\text { Factors } \\
\text { Associated with } \\
\text { DV }\end{array}$ & $\begin{array}{l}\text { Individual, } 934 \\
\text { women visiting } \\
\text { OB/GYN clinics. } \\
\text { Study controlled } \\
\text { for religion for } \\
\text { prevalence } \\
\text { rates }\end{array}$ & $\begin{array}{l}\text { Quantitative, } \\
\text { Primary, } \\
\text { Systematic } \\
\text { random } \\
\text { sampling }\end{array}$ & $\begin{array}{l}\text { Structured questionnaire } \\
\text { using existing scales } \\
\text { Not discussed as they used } \\
\text { established scales }\end{array}$ & $\begin{array}{l}\text { Random sampling increases } \\
\text { generalizability } \\
\text { Addresses an important topic } \\
\text { but no discussion of validity and } \\
\text { reliability }\end{array}$ \\
\hline $\begin{array}{l}\text { Haj-Yahia, } \\
\text { M. M. \& } \\
\text { Uysal, A }\end{array}$ & $\begin{array}{l}\text { Beliefs about Wife } \\
\text { Beating among Medical } \\
\text { Students from Turkey, } \\
2008\end{array}$ & Turkey & $\begin{array}{l}\text { Attitudes Towards } \\
\text { DV; Service } \\
\text { Response }\end{array}$ & $\begin{array}{l}\text { Individual, } 173 \\
\text { Turkish medical } \\
\text { students. } \\
\text { Over } 90 \% \text { of } \\
\text { participants are } \\
\text { Muslim }\end{array}$ & $\begin{array}{l}\text { Quantitative, } \\
\text { Primary, } \\
\text { Convenience } \\
\text { sampling }\end{array}$ & $\begin{array}{l}\text { Used existing measures } \\
\text { Reliability discussed, not } \\
\text { validity }\end{array}$ & $\begin{array}{l}\text { Primary data collection; retested } \\
\text { the reliability of existing measures } \\
\text { Validity was not tested } \\
\text { Limited generalizability due to } \\
\text { convenience sampling }\end{array}$ \\
\hline $\begin{array}{l}\text { Abugideiri, } \\
\text { S. }\end{array}$ & $\begin{array}{l}\text { Domestic Violence among } \\
\text { Muslims Seeking Mental } \\
\text { Health Counseling, } 2007\end{array}$ & Unite States & Prevalence & $\begin{array}{l}\text { Individual, } 190 \\
\text { Muslim mental } \\
\text { health clients }\end{array}$ & $\begin{array}{l}\text { Quantitative, } \\
\text { Primary, } \\
\text { convenience } \\
\text { sampling }\end{array}$ & $\begin{array}{l}\text { Retrospective client records } \\
\text { No discussion of validity } \\
\text { and reliability }\end{array}$ & $\begin{array}{l}\text { Important topics covered } \\
\text { No discussion of validity and } \\
\text { reliability } \\
\text { Convenience sample limits } \\
\text { generalizability }\end{array}$ \\
\hline $\begin{array}{l}\text { Abu-Ras, } \\
\text { W. }\end{array}$ & $\begin{array}{l}\text { Cultural Beliefs and } \\
\text { Service } \\
\text { Utilization by Battered } \\
\text { Arab Immigrant Women, } \\
2007\end{array}$ & Unite States & $\begin{array}{l}\text { Factors } \\
\text { Associated with } \\
\text { DV; Experiences } \\
\text { of Coping and } \\
\text { Support }\end{array}$ & $\begin{array}{l}\text { Individual, } 86 \\
\text { Muslim, Arab } \\
\text { immigrant } \\
\text { women- } \\
\text { individual }\end{array}$ & $\begin{array}{l}\text { Quantitative, } \\
\text { Primary, } \\
\text { Purposeful } \\
\text { sampling }\end{array}$ & $\begin{array}{l}\text { Existing scales on: of } \\
\text { abuse, help-seeking } \\
\text { behavior, and attitudes } \\
\text { toward abuse } \\
\text { Pilot study, and panel of } \\
\text { judges critique the scales }\end{array}$ & $\begin{array}{l}\text { Extremely useful study } \\
\text { Purposeful sampling limits } \\
\text { generalizability, uses validity } \\
\text { and reliability but extensive } \\
\text { revalidation of scales were not } \\
\text { conducted }\end{array}$ \\
\hline $\begin{array}{l}\text { Adam, N. M. } \\
\text { \& Schewe, } \\
\text { P. }\end{array}$ & $\begin{array}{l}\text { Multilevel Framework } \\
\text { Exploring Domestic } \\
\text { Violence Against } \\
\text { Immigrant Indian } \\
\text { and Pakistani Women in } \\
\text { the United States, } 2007\end{array}$ & Unite States & $\begin{array}{l}\text { Factors } \\
\text { Associated with } \\
\text { DV }\end{array}$ & $\begin{array}{l}\text { Individual, } 78 \\
\text { Indian and } \\
\text { Pakistani } \\
\text { women ( } 41 \\
\text { Muslim). } \\
\text { Controlled for } \\
\text { religion for } \\
\text { prevalence }\end{array}$ & $\begin{array}{l}\text { Quantitative, } \\
\text { Primary, } \\
\text { Purposeful } \\
\text { sampling }\end{array}$ & $\begin{array}{l}\text { Surveys } \\
\text { Reliability tested, not } \\
\text { validity of scales }\end{array}$ & $\begin{array}{l}\text { Nationwide sampling, controlled } \\
\text { for religion, non-random sampling } \\
\text { limits generalizability, only } \\
\text { reliability tested }\end{array}$ \\
\hline Yount, K. M. & $\begin{array}{l}\text { Resources, Family } \\
\text { Organization, and } \\
\text { Domestic Violence } \\
\text { Against Married Women } \\
\text { in Minya, Egypt, } 2005 .\end{array}$ & Egypt & $\begin{array}{l}\text { Factors } \\
\text { Associated with } \\
\text { DV }\end{array}$ & $\begin{array}{l}\text { Individual, } 2522 \\
\text { married women } \\
\text { in Minya, Egypt } \\
\text { Religion was a } \\
\text { control variable. } \\
77 \% \text { were } \\
\text { Muslim. }\end{array}$ & $\begin{array}{l}\text { Quantitative, } \\
\text { Secondary }\end{array}$ & $\begin{array}{l}\text { Two national government } \\
\text { surveys } \\
\text { No discussion of validity } \\
\text { and reliability }\end{array}$ & $\begin{array}{l}\text { National representative sample } \\
\text { that used cluster sampling } \\
\text { increases generalizability, } \\
\text { however no discussion of } \\
\text { reliability and validity of measures }\end{array}$ \\
\hline $\begin{array}{l}\text { Almosaed, } \\
\text { N. }\end{array}$ & $\begin{array}{l}\text { Violence Against } \\
\text { Women: A Cross-cultural } \\
\text { Perspective, } 2004\end{array}$ & $\begin{array}{l}\text { Saudi } \\
\text { Arabia }\end{array}$ & $\begin{array}{l}\text { Prevalence; } \\
\text { Attitudes Towards } \\
\text { DV }\end{array}$ & $\begin{array}{l}\text { Individual, } 230 \\
\text { Muslim men } \\
\text { and women } \\
\text { living in Jeddah. } \\
99 \% \text { were } \\
\text { Muslim }\end{array}$ & $\begin{array}{l}\text { Quantitative, } \\
\text { Primary, } \\
\text { Snowball } \\
\text { sampling }\end{array}$ & $\begin{array}{l}\text { Two opinion survey } \\
\text { questionnaires (one for } \\
\text { men, one for women) } \\
\text { created by the author } \\
\text { No discussion of validity } \\
\text { and reliability }\end{array}$ & $\begin{array}{l}\text { Discussed an important topic } \\
\text { with significant findings, however } \\
\text { validity and reliability not } \\
\text { discussed } \\
\text { Due to non-random sampling } \\
\text { generalizability is limited }\end{array}$ \\
\hline $\begin{array}{l}\text { Abu-Ras, } \\
\text { W. }\end{array}$ & $\begin{array}{l}\text { Barriers to services for } \\
\text { battered Arab immigrant } \\
\text { women in a Detroit } \\
\text { suburb, } 2003\end{array}$ & Unite States & $\begin{array}{l}\text { Experiences } \\
\text { of Coping and } \\
\text { Support }\end{array}$ & $\begin{array}{l}\text { Individual, } 67 \\
\text { Arab immigrant } \\
\text { women }(85.1 \% \\
\text { Muslim) }\end{array}$ & $\begin{array}{l}\text { Quantitative, } \\
\text { Primary, } \\
\text { Purposeful } \\
\text { sampling }\end{array}$ & $\begin{array}{l}\text { Cross-sectional survey } \\
\text { collected using in-depth } \\
\text { interviews }\end{array}$ & $\begin{array}{l}\text { Important topic, future studies } \\
\text { can benefit from utilizing mix } \\
\text { methods; generalizability is } \\
\text { limited }\end{array}$ \\
\hline
\end{tabular}




\begin{tabular}{|c|c|c|c|c|c|c|c|}
\hline $\begin{array}{l}\text { Cwikel, J., } \\
\text { Lev-Wiesel, } \\
\text { R. \& Al- } \\
\text { Krenawi, A. }\end{array}$ & $\begin{array}{l}\text { The Physical and } \\
\text { Psychosocial Health of } \\
\text { Bedouin Arab Women of } \\
\text { the Negev Area of Israel: } \\
\text { The Impact of High } \\
\text { Fertility and Pervasive } \\
\text { Domestic Violence, 2003. }\end{array}$ & Israel & $\begin{array}{l}\text { Prevalence } \\
\text { and Type of } \\
\text { Abuse; Health } \\
\text { Consequences } \\
\text { and DV }\end{array}$ & $\begin{array}{l}\text { Individual, } 202 \\
\text { Bedouin Arab } \\
\text { Muslim women }\end{array}$ & $\begin{array}{l}\text { Quantitative, } \\
\text { Primary, } \\
\text { Quota } \\
\text { sampling }\end{array}$ & $\begin{array}{l}\text { Uses established scaling. } \\
\text { Not discussed as they used } \\
\text { established scales }\end{array}$ & $\begin{array}{l}\text { Quota sampling increases } \\
\text { generalizability when random } \\
\text { sampling is not possible } \\
\text { Validity and reliability discussed }\end{array}$ \\
\hline $\begin{array}{l}\text { Haj-Yahia. } \\
\text { M }\end{array}$ & $\begin{array}{l}\text { Beliefs about wife beating } \\
\text { among Arab men from } \\
\text { Israel: influence of their } \\
\text { patriarchal ideology, } \\
2003\end{array}$ & Israel & $\begin{array}{l}\text { Attitudes Towards } \\
\text { DV }\end{array}$ & $\begin{array}{l}\text { Individual, } 362 \\
\text { Arab men. } 82 \% \\
\text { were Muslim }\end{array}$ & $\begin{array}{l}\text { Quantitative, } \\
\text { Primary, } \\
\text { Systematic } \\
\text { random } \\
\text { sampling }\end{array}$ & $\begin{array}{l}\text { Existing scales and ones } \\
\text { created by the author } \\
\text { Tested reliability and } \\
\text { validity }\end{array}$ & $\begin{array}{l}\text { Used random sampling limits } \\
\text { generalizability, reliability was } \\
\text { tested for all instruments, one of } \\
\text { the few studies looking at male } \\
\text { perspective, including additional } \\
\text { variables can benefit the study }\end{array}$ \\
\hline $\begin{array}{l}\text { Haj-Yahia, } \\
\text { M. }\end{array}$ & $\begin{array}{l}\text { Attitudes of Arab women } \\
\text { towards different patterns } \\
\text { of coping with wife abuse, } \\
2002\end{array}$ & Israel & $\begin{array}{l}\text { Experiences } \\
\text { of Coping and } \\
\text { Support; Attitudes } \\
\text { Towards DV }\end{array}$ & $\begin{array}{l}\text { Individual, } 291 \\
\text { Arab women } \\
\text { (79\% of the } \\
\text { participants } \\
\text { were Muslim) }\end{array}$ & $\begin{array}{l}\text { Quantitative, } \\
\text { Primary, } \\
\text { Convenience } \\
\text { sampling }\end{array}$ & $\begin{array}{l}\text { Existing scales and ones } \\
\text { created by the author } \\
\text { Tested reliability but validity } \\
\text { was not discussed }\end{array}$ & $\begin{array}{l}\text { Looked at an important area, } \\
\text { however non-random sampling } \\
\text { limits the generalizability of the } \\
\text { study, only reliability was tested }\end{array}$ \\
\hline $\begin{array}{l}\text { Ali, A. \& } \\
\text { Toner, B. B. }\end{array}$ & $\begin{array}{l}\text { Self-esteem as a predictor } \\
\text { of attitudes toward wife } \\
\text { abuse among Muslim } \\
\text { women and men in } \\
\text { Canada, } 2001\end{array}$ & Canada & $\begin{array}{l}\text { Attitudes Towards } \\
\text { DV }\end{array}$ & $\begin{array}{l}\text { Individual, } 40 \\
\text { Muslim men } \\
\text { and women }\end{array}$ & $\begin{array}{l}\text { Quantitative, } \\
\text { Primary, } \\
\text { Convenience } \\
\text { sampling }\end{array}$ & $\begin{array}{l}\text { Use of standardized } \\
\text { measures } \\
\text { Tested reliability not validity }\end{array}$ & $\begin{array}{l}\text { Important topic } \\
\text { However, methodology and } \\
\text { analysis were not clearly } \\
\text { specified }\end{array}$ \\
\hline $\begin{array}{l}\text { Koening, M., } \\
\text { Hossain, M. } \\
\text { B., Ahmed, } \\
\text { S., \& } \\
\text { Haaga, J. }\end{array}$ & $\begin{array}{l}\text { Individual and community } \\
\text { determinants of domestic } \\
\text { violence in rural } \\
\text { Bangladesh } 1999\end{array}$ & Bangladesh & $\begin{array}{l}\text { Prevalence and } \\
\text { Type of Abuse; } \\
\text { Experiences } \\
\text { of Coping and } \\
\text { Support }\end{array}$ & $\begin{array}{l}\text { Individual, } \\
\text { 10,368 rural } \\
\text { Bangladeshi, } \\
\text { majority Muslim }\end{array}$ & $\begin{array}{l}\text { Quantitative, } \\
\text { Primary, } \\
\text { Purposeful } \\
\text { sampling }\end{array}$ & $\begin{array}{l}\text { Survey methods with two } \\
\text { study sites } \\
\text { No discussion of validity } \\
\text { and reliability }\end{array}$ & $\begin{array}{l}\text { Large sample size, however, } \\
\text { non-random sampling limits } \\
\text { generalizability, reliability and } \\
\text { validity were not discussed }\end{array}$ \\
\hline $\begin{array}{l}\text { Kulwicki, A. } \\
\text { D. \& Miller, } \\
\text { J. }\end{array}$ & $\begin{array}{l}\text { Domestic violence in } \\
\text { the Arab American } \\
\text { population: Transforming } \\
\text { environmental conditions } \\
\text { through community } \\
\text { education, } 1999\end{array}$ & Unite States & $\begin{array}{l}\text { Attitudes Toward } \\
\text { DV; Service } \\
\text { Response }\end{array}$ & $\begin{array}{l}\text { Individual, } \\
202 \text { Muslim, } \\
\text { Arab American } \\
\text { immigrants }\end{array}$ & $\begin{array}{l}\text { Quantitative, } \\
\text { Primary, } \\
\text { Convenience } \\
\text { sampling }\end{array}$ & $\begin{array}{l}\text { Surveys } \\
\text { Survey tools were pilot } \\
\text { tested but validity and } \\
\text { reliability was not formally } \\
\text { discussed }\end{array}$ & $\begin{array}{l}\text { Important topic using action } \\
\text { research design, limited } \\
\text { generalizability }\end{array}$ \\
\hline
\end{tabular}

Table 1: Sample Characteristics.

\section{Results}

\section{Sample characteristics}

Of the 35 studies (Table 1) that are being used for this review, 13 were conducted in the US and 22 were done globally. Other countries studied include Bangladesh (4), Israel (4), Jordan (3), Egypt (2), Nigeria (1), Canada (2), Palestine (1), Germany (1), Turkey (1), Kazakhstan (1), Saudi Arabia (1), and Lebanon (1). Fifteen studies concentrated exclusively on Muslims and 10 of the studies included in the analysis controlled for religion with implications discussed in detail. In 10 studies, at least $75 \%$ of participants were of Muslim background. Nineteen studies focused on women while 15 studied both men and women, and one study targeted men only. The unit of analysis for all but one of the 35 studies was at the individual level with that being at the focus group level. Sample sizes varied from 2 to nearly 11,000 participants. Thirty-one studies used primary data, while 4 studies used secondary data. Of the studies that used primary data, 3 studies used random sampling methods, 28 used non-random sampling methods and one mixed-method study used both random and non-random sampling on their two datasets. Of the 4 studies that used secondary data, the data were obtained from large government health surveys. Seven studies used existing scales and 9 studies used scales created by the authors. Six studies used semi-structured interviews whereas 4 studies chose an unstructured interview format.One study reviewed client records while another study combined a review of police records with a semi-structured interview. Finally, one study used field observations.

\section{Summary of the Quality Assessment}

The study findings from our assessment are presented in Table 2.
Overall studies varied in their rigor. Qualitative studies are stronger in their rigor than quantitative studies. Among quantitative studies, only 3 studies were ranked strong in all areas of assessment and 3 studies were ranked weak overall. The other 16 studies varied in their ranking based on criteria assessment. The strongest area of the quantitative studies was in their interpretation of results. Fifteen studies are ranked strong in this theme. The biggest bias in the studies is identified in the area of generalizability. Twelve studies used non-probability sampling methods reducing the generalizability. Qualitative studies were higher in their rigor comparatively in methodology overall. Out of the 11 qualitative studies, 4 are ranked strong in all areas, and none are ranked weak in all areas. Qualitative studies are clearly outlined their study, in fact 10 of the 11 studies are ranked strong. The weakest areas of qualitative studies are in transferability and measurement error. The two mixed methods studies are ranked overall weak to moderate in their rigor (Table 2).

\section{Themes}

Some major themes stood out in the articles: prevalence and types of abuse, factors associated with IPV, attitudes towards IPV, experiences of coping and support, IPV and health consequences, and service response.

Prevalence and types of abuse: Extremely few studies have attempted to examine the prevalence of IPV in Muslim communities. Of the studies available, high global prevalence rates are reported overall. The quality assessment of these studies, however, reveals weak study rigor, therefore it is difficult to make definitive conclusions and generalizability is considered to be low. It is also difficult to infer if the 
Citation: Jayasundara D, Nedegaard R, Sharma B, Flanagan K (2014) Intimate Partner Violence in Muslim Communities. Arts Social Sci J S1: 003. doi: 10.4172/2151-6200.S1-003

Page 8 of 12

\begin{tabular}{|c|c|c|c|c|c|}
\hline Author/s & $\begin{array}{l}\text { Clearly outlined } \\
\text { Study design }\end{array}$ & $\begin{array}{l}\text { Measurement error } \\
\text { (validity and reliability) }\end{array}$ & $\begin{array}{l}\text { Appropriateness } \\
\text { of analysis }\end{array}$ & $\begin{array}{l}\text { Interpretation } \\
\text { of Results }\end{array}$ & $\begin{array}{l}\text { Generalizability/ } \\
\text { transferability }\end{array}$ \\
\hline \multicolumn{6}{|l|}{ Qualitative } \\
\hline Zakar, R., Zakar, M. Z., Faist, T. \& Kraemer, A., 2012 & Strong & Strong & Strong & Moderate & Moderate \\
\hline Desai, S. \& Haffajee, Z., 2011 & Weak & Weak & Moderate & Moderate & Weak \\
\hline $\begin{array}{l}\text { Kulwicki, A., Hymes, R., Hammad, A., Killawi, A., \& Farrag, } \\
\text { M., } 2010\end{array}$ & Strong & Strong & Strong & Strong & Moderate \\
\hline GharaibeH, M., \& Oweis, A., 2009 & Strong & Moderate & Strong & Strong & Strong \\
\hline Snajdr, E., 2005 & Strong & Weak & Weak & Moderate & Weak \\
\hline Hassouneh-Phillips, D. 2003 & Strong & Strong & Strong & Strong & Strong \\
\hline Hassouneh-Phillips, D. 2001a & Strong & Strong & Strong & Strong & Strong \\
\hline Hassouneh-Phillips, D. S 2001b & Strong & Strong & Strong & Strong & Strong \\
\hline Hassouneh-Phillips, D., 2000 & Strong & Strong & Strong & Strong & Strong \\
\hline Ammar, N. H., 2000 & Strong & Strong & Strong & Strong & Moderate \\
\hline Keenan, C. K., El-Hadad, A. \& Balian, S. A., 1998 & Strong & Strong & Strong & Strong & Moderate \\
\hline \multicolumn{6}{|l|}{ Mixed Methods } \\
\hline Btoush, R. \& Haj-Yahia, M. M., 2008 & Moderate & Weak & Weak & Moderate & Weak \\
\hline Haj-Yahia, M., 2000 & Moderate & Weak & Weak & Weak & Weak \\
\hline \multicolumn{6}{|l|}{ Quantitative } \\
\hline Dalal, K., Andrews, J. \& Dawad, S., 2011 & Moderate & Weak & Strong & Strong & Strong \\
\hline Haj-Yahia, M. M., Wilson, R. M. \& Naqvi, S. A. M., 2012 & Strong & Strong & Strong & Strong & Strong \\
\hline $\begin{array}{l}\text { Rahman, M., Sasagawa, T., Fujii, R, Tomizawa, H. \& Makinoda, } \\
\text { S., } 2012\end{array}$ & Moderate & Weak & Strong & Strong & Strong \\
\hline Celik, A. \& Sabri, B., 2011 & Weak & Weak & Weak & Weak & Weak \\
\hline $\begin{array}{l}\text { Haddad, L. G., Shotar, A., Younger, J. B., Alzyoud, S. \& } \\
\text { Bouhaidar, C. M., } 2011\end{array}$ & Strong & Strong & Strong & Strong & Strong \\
\hline Yount, K.M. \& Li, L., 2010 & Moderate & Weak & Moderate & Strong & Strong \\
\hline Dalal, K., Raman, R. \& Jansson, B., 2009 & Moderate & Weak & Strong & Strong & Strong \\
\hline Ghayyur, T., 2009 & Weak & Weak & Weak & Weak & Weak \\
\hline Okenwa, L. E., Lawoko, S. \& Jansson, B., 2009 & Moderate & Weak & Strong & Moderate & Strong \\
\hline Haj-Yahia, M. M. \& Uysal, A, 2008 & Strong & Moderate & Strong & Strong & Weak \\
\hline Abugideiri, S, 2007 & Weak & Weak & Weak & Weak & Weak \\
\hline Abu-Ras, W., 2003 & Strong & Moderate & Weak & Strong & Weak \\
\hline Adam, N. M. \& Schewe, P., 2007 & Strong & Strong & Strong & Strong & Weak \\
\hline Yount, K. M., 2005 & Moderate & Strong & Strong & Strong & Strong \\
\hline Almosaed, N., 2004 & Weak & Weak & Weak & Weak & Weak \\
\hline Abu-Ras, W., 2007 & Strong & Strong & Strong & Strong & Weak \\
\hline Cwikel, J., Lev-Wiesel, R. \& Al-Krenawi, A., 2003 & Strong & Moderate & Moderate & Strong & Moderate \\
\hline Haj-Yahia. M, 2003 & Strong & Strong & Strong & Strong & Strong \\
\hline Haj-Yahia, M., 2002 & Strong & Strong & Strong & Strong & Weak \\
\hline Ali, A. \& Toner, B. B., 2001 & Moderate & Strong & Weak & Weak & Weak \\
\hline Koening, M., Hossain, M. B., Ahmed, S., \& Haaga, J., 1999 & Moderate & Weak & Strong & Strong & Weak \\
\hline Kulwicki, A. D. \& Miller, J., 1999 & Moderate & Weak & Weak & Moderate & Weak \\
\hline
\end{tabular}

Table 2: Summary of quality assessment.

variations in prevalence rates are due to the way abuse was measured, the rigor of these studies, or due to cultural and national variations.

Three US-based studies are identified that examine prevalence. While these studies did not use rigorous research designs, they are the only available records of prevalence in the US. A third noteworthy study done by Alkhateeb [22] is unfortunately excluded in this study due to a lack of information on the methods she used. These studies found that $66-70 \%$ of subjects reported that they knew a victim of IPV. Between 26 and $73 \%$ of the women surveyed reported having experiencing some form of IPV either at the time of the survey or during their lifetime. Roughly, 52\% felt that IPV in America is increasing, and about $65 \%$ of the respondents felt that IPV was a major problem in their community. More women in the survey (72.49\%), than men (33.93\%) identified IPV as a major issue in their community. However, less than half of the respondents knew of an IPV shelter in their community and $82 \%$ believed the mosques were not doing enough to deal with IPV [29-
31] compared immigrant Indian and Pakistani women living in the US.While the primary focus was not on the incidence rates with the Muslim population, this study did control for religion (subjects were either Muslims or Hindus) and found Hindu (81\%) participants to be more likely to be victims of abuse in their lifetimes than Muslims (72\%). Unfortunately, due to low rigor in both studies the conclusions drawn cannot be considered definitive.

These high prevalence rates were also echoed globally. One study conducted in Israel with Bedouin Arab women identified that $48 \%$ reported a lifetime exposure to physical violence, with women younger than $40(33.5 \%)$ being more likely to be abused than women over $40(21.3 \%)$ [32]. Their findings also indicated that women were less likely to face abuse when they were pregnant. Haddadet al. [33] conducted a study in Jordan and found that $39 \%$ of the participating women reported emotional abuse, while $30 \%$ reported physical abuse and $6 \%$ reported sexual abuse. Three studies conducted in Bangladesh 
identified that Muslim women were more likely to experience all forms of IPV than their non-Muslim counterparts [34-36]. However, a fourth study by Dalalet al. [37] found the same differences between Muslim and non-Muslim women in all forms of abuse except for physical abuse. Contrary to the findings of the Bangladeshi studies, research conducted with Lebanese women found Armenian women in the study cohort reporting more physical abuse than Muslim women [38]. Yet another study conducted in Nigeria reported that Catholic women experienced significantly higher sexual abuse than Muslim women. No comparable significant differences were identified with physical and psychological abuse between Muslim participants and their Catholic counterparts [39]. Consistent with the Bangladeshi studies, an Egyptian study also found Muslim women to have higher prevalence levels of IPV (18.4\%) compared to their Christian counterparts (14\%) [40]. A Jordanian study on wife abuse targeted both men and women and identified that $96 \%$ of the study participants reported some form of verbal and emotional wife abuse. Sixty-one percent of the participants reported mild to severe physical wife abuse, $5 \%$ reported acts that were classified as sexual abuse, and only $1 \%$ reported acts that could be categorized as economic abuse [41]. Finally, a study conducted in Saudi Arabia comparing men and women found that $30 \%$ of the participating men were physically abusive towards their wives, while $34 \%$ of the women identified themselves as victims. Additionally, this study found that $41 \%$ of the participants knew a family member who was also a victim [42]. Once again, limitations of these studies threaten their validity and implications.

Other studies found several key types of IPV. Unique features of these include public humiliation, insults and ridicule of the wife, and engaging in abusive behavior in front of the in-laws and neighbors [43]; misuse of religion to be a specific form of abuse [44]; and misuse of polygamy as a form of partner abuse where participants in the study found that the husband's polygamy misuse as problematic, not polygamy itself.Based on this study polygamy comes with a set of expected behaviors by all parties involved and when the husband deviated from the Islamic dictates of polygamous marriage, such as unequal treatment of wives, the women in this study identified it as a form of emotional abuse.Co-wives were identified as both witnesses to abuse as well as co-perpetrators [16]. While these findings are interesting, there is an insufficient number of studies available to make conclusions based on culture and nationality.

Factors associated with intimate partner violence: A total of two studies examined factors associated with IPV and validated scales measuring attitudes, risk factors, and culturally sensitive screening. One study measured IPV risk among Arabic women [15] and found that "religious beliefs, family support, economic, social, immigration status, legal rights and English language skills" were critical for risk assessment (p. 12). Haddad et al. [33] was able to demonstrate initial reliability and validity of an Arabic language version of the NorVold Domestic Abuse Questionnaire (NORAQ) to successfully screen IPV in Jordan. The order of factors identified was similar to original scale. Several qualitative studies discussed interlocking factors such as immigration stressors, isolation, spirituality, racism, sexism, work related stressors, conflict with the husband's family, financial stressors, and alcohol abuse by husband as associated with IPV [38,44-46]. These studies revealed and emphasized the complexity IPV situations often have when additional factors are introduced.

Attitudes towards intimate partner violence: Nine studies looked at attitudes toward intimate partner violence [41-43,46-51]. A tenth study on a social work practice scale to measure an Arab American women's definition of IPV was not included in this study due to the fact that this dissertation was not available to the public per author request. Generally, studies looking at attitudes towards IPV examined tendencies to justify wife abuse especially under certain circumstances and especially by men, regardless of the cultural and global context.One study conducted in the US on Arab American men and women found that over $58 \%$ of the women and $59 \%$ of the men thought it was okay to slap a wife if she hits the husband first. However, surprisingly $48 \%$ of the women and $23 \%$ of the men thought it was okay for the husband to hit his wife if she has an affair with $18 \%$ of the women justifying homicide if a wife has an affair [51]. A Canadian study also reported significantly more lenient attitudes towards wife abuse by Muslim men than Muslim women. However, this study found those negative attitudes were mitigated by higher self-esteem, where Muslims with higher selfesteem were more likely to be against wife abuse, regardless of gender [47]. A study conducted in Germany on immigrant Pakistani women found that some women in the study believed IPV is not a problem in their community, that the idea of abuse as a crime is a western construct and serves to break the family institution. Some even believed that a certain level of abuse is justified and necessary for a marriage [46] Hassouneh-Phillips [20] found that American Muslim women in her study identified marriage as the center of social and religious life and that it was a significant source of personal and spiritual fulfillment. Additionally, women were socialized at a very young age to one day marry, thus substantial pressure from society is exerted to get married and stay married. This leads to significant acceptance and tolerance of wife abuse. Additionally, the belief that cultural and religious mores dictate that good wives are obedient means that women would not question male authority or fight back, thus leading to the belief that a good wife should be tolerant of abuse or that the husband has right to abuse. Many women believed in total submission to Allah, and believed that submission to her husband's wishes were an extension of this.

Experiences of coping and support: This review indicates that Muslim women who experienced IPV coped with it by attempting to tolerate it, taking responsibility of their victimization, attempting to change their behavior, taking responsibility to change their husband's behavior, utilizing indigenous methods and seeking support from informal agents of support $[36,46]$. Experiences of coping and help seeking were strongly tied to cultural and religious contexts and beliefs as Muslim women living in North America come from various cultural national backgrounds $[8,17,18,20,44]$. Spirituality in these studies was strongly tied to cultural beliefs and played a complex role as it could serve both to provide strength as a source of coping, or create vulnerability for continued IPV as a mechanism of obstruction for safety $[8,20,44]$. For example Hassouneh-Phillips [20] found a process she calls "negation of self" among the American Muslim women in her study. Living by what they perceive the society dictates can lead to attempts to change self and take responsibility of their partners' abuse.

Additionally, two other studies, one in Jordan and one among immigrant Pakistani women in Germany, found that victims' decisions to live in abusive relationships were justified by one's inherited social background, lack of social support, sacrificing self for one's children, stigma and social consequences associated with divorce, a belief that one's quality of life would be worse, not being able to remarry, having to return back to one's parents' home, belief in the privacy of the marriage institution, economic dependency, an unappealing cost and benefit analyses, and the rejection of idea that the abuse is a serious problem $[21,46]$.

Zakaret al. [46] studied immigrant Pakistani women living in 
Germany and found the following indigenous strategies to cope with and mitigate tension the most helpful:avoiding conflict, reconfiguring the relationship, using the power of silence, practicing patience, and contacting one's family back home for advice and support. Koeninget al. [36] studied Bangladeshi women and found that negotiating with one's partner, taking initiative, attempting to appease, and passively resigning were the methods most used by study participants. Studies also found that the two most often used support systems were seeking assistance from in-laws and seeking assistance from one's own family. Women were more likely to seek assistance from their own family if they faced severe abuse while they generally sought out in-laws if abuse was occasional $[41,36]$.

Seeking help from formal systems and leaving were the least utilized supports among several studies globally. Haj-Yahia [43], studied women who held strong views against intimate partner violence, nevertheless felt that when it comes to help seeking, Arab cultural mores should be maintained. They believed that coping with abuse should respect cultural values, maintain family privacy, and be discreet. They also believed the family should be the first help-seeking option. Haj-Yahia [52], found that less than 1 in 3 women sought outside help for physical abuse; however, the severity of the abuse helped predict the likelihood of seeking assistance from outside. Zakaret al. [46] identified several obstacles for help-seeking from formal systems: inability to understand the structure and approaches of formal care systems, lack of trust that they will actually help, fear of consequences (especially from their abusive partners), and inability the explain problems. A strong desire to not break the marriage by leaving was reflected in several studies $[8,19,20,46,52]$. A study conducted in the US on Muslim women's experiences of leaving abusive relationships revealeda multistep, complex process involving four stages: "reaching the point of saturation, getting khula (Islamic divorce initiated by wives), facing family and/or community disapproval, and reclaiming the self" [19].

IPV and associated health issues: Overall, studies that looked at the association between IPV and health conditions found various negative health conditions among women undergoing IPV, indicating that IPV impacts all aspects of victims' lives. In a recent survey conducted in the US, the most often identified mental health effects of partner abuse were: depression, low self-esteem, shame, helplessness, hopelessness and anxiety. The physical symptoms associated with IPV included: bruises, body pain, nausea and vomiting [30]. Similar health related patterns were identified in studies conducted abroad. Cwikelet al. [32] conducted a study of Muslims in Israel and found significant partial correlations between the following health problems and IPV: gynecological problems, reporting any illness, poor self-esteem, symptoms of hopelessness, and/or helplessness, and other symptoms of depression. In addition, IPV was associated with a higher number of children. Rahman et al. [37] conducted a study in Bangladesh that mirrors this finding. They found Muslim women to be significantly more likely to experience unintended pregnancies than non-Muslim women. However, a study by Dalalet al. [34] on contraception use and IPV targeting both Muslim and non-Muslim women in Bangladesh found that abused women are twice as likely to use contraceptives. They found physical abuse is significantly associated with contraceptive use, while sexual abuse is not. More importantly no significant differences are identified between exposure to IPV and contraceptive use between Muslim and non-Muslim women.

Service provider's perceptions of abuse: Four studies were related to service response to IPV $[49,51,53,54$,]. Two studies were based in the US. The action research study based on critical theory by
Kulwicki and Miller [51] assessed the attitudes towards IPV in an Arab American community and implemented local strategies accordingly to combat wife abuse. They conclude that any intervention approaches implemented with Muslim communities must take into consideration the implications of traditional gender ideologies and behavior towards IPV. Consequently, their project led to active IPV awareness campaigns through media and outreach work in the communities [51]. A second paper looked at police response to IPV in Muslim Communities in the US [53]. More specifically, this study examined the "interplay between diversity in a group's identity and imposition of simplified stereotyping in policing IPV in an Arab American community" [53] found that the Arab identities within the community have great diversity. Consequently, stereotypic thinking by the police can lead to potentially dire consequences for Arab American families experiencing IPV. One study [54] from Kazakhstan looked at a Muslim grass roots agency's response and how local grassroots level groups were helping women undergoing IPV by using local cultural and religious ideologies of the community to guide the victim's choices, without compromising the agency's own goals. They separated themselves from western and larger granters to help clients in ways they deemed culturally appropriate. A final study discussed IPV service response from the perspective of healthcare. Haj-Yahia and Uysal [49] found that while 5-39\% of medical students in Turkey justified wife beating, most (68-91\%) expressed a willingness to help the victims. Fewer (28.5\%) believed it was the women's responsibility for the beating as about $50 \%$ held the husband responsible.Surprisingly, only $25 \%$ supported punishing male IPV perpetrators and a small minority of the students even believed that the wife somehow benefits from the abuse.

\section{Discussion}

It is extremely important to understand IPV dynamics among Muslim communities and their variations.As a first step towards this end, this study conducted a systematic review. The primary focus of this study was to develop an overview of the findings of the past studies that empirically examined the topic of IPV and Muslims globally. Study findings indicated that the articles looked at the following themes: prevalence and types of abuse, factors associated with IPV, attitudes towards IPV, experiences of coping and support, IPV and health consequences, and service response. In general these studies found high prevalence levels of IPV among Muslims living in the US and globally. Consistent with IPV research in general, verbal and psychological abuse was found most prevalent, followed by physical, sexual, and fatal. Individual family, and interlocking social problems, traditional beliefs, spirituality, and immigration-related stressors were associated with IPV as identified by several studies.Studies on beliefs about IPV found that there tended to be higher leniency and justification of abuse in Muslim cultures than is the Western norm. Muslim men across the board tended to justify abuse more than Muslim women, except when the wife had an affair. Then both men and women held more negative views, with women justifying abuse in some studies even more than men. Beliefs were interwoven with patriarchal and traditional gender roles. These same beliefs created unique coping contexts for women, where they were more likely to use indigenous methods and seek help from informal agents, rather than seek help from formal mechanism or leave. Consequently experiences of coping and help seeking were strongly tied to cultural and religious contexts of their beliefs.

Except for few studies the majority of the quantitative studies did not use rigorous methodologies. Due to these limitations, generalizability of these studies is low. However, given the hidden nature of the topic, the exploratory nature of these studies was not surprising. Qualitative 
studies presented more rigorous overviews. Once again as this is both difficult and an understudied area globally, qualitative methodologies are more appropriate for topics of this nature to obtain preliminary, yet, in-depth understanding of the topic area.

Past studies have left several gaps, and given the non-rigorous nature of prevalence studies, it is extremely difficult to summarize conclusively the prevalence rates of domestic violence.The authors attempted to obtain a clearer picture of IPV in Muslim communities from a global perspective for North American social scientists. However, these studies are in no way sufficient in gaining a more comprehensive understanding of the totality of the problem.Thus rigorous studies are needed both in North America and globally. Comparative studies across countries and continents will also benefit to identify prevalence levels as well as the nature and impact on various Muslim communities.

The impact that cultural variations have on IPV has not adequately been captured.In fact, the attempt to synthesize the current literature may inadvertently diminish the numerous variations within Muslim culture.These studies did not examine the direct impact of the religion to help identify protective factors and/or vulnerabilities and have not adequately examined the impact current interventions have on this population. Not only is the knowledge base inadequate for scholars and social scientists, but advocates and practitioners would benefit from a more comprehensive way of understanding religious and cultural aspects as well.This would allow them to provide services that better address the unique needs of Muslims victims.Additionally, important factors such as the impact of $9 / 11$ on Muslim families related to IPV, have not been addressed in this literature. These all leave implications for future studies. There is a greater need for further study in this area, especially on the intersections of Islam, culture, political history, socioeconomic status, immigration status and the history of patriarchal oppression related to cultural practices of families.Additionally, how these factors relate to competent assessment of service needs, barriers to receiving services and successful service delivery requires further study.Consequently, well-rounded studies that attempt to localize knowledge by looking at all aspects of service delivery as they relate to IPV among Muslim communities are needed. More studies are also needed looking at success of efforts taken by advocates to educate communities globally, the role played by religious leaders (Imams) and men combating IPV situations are especially absent in past studies and need to addressed.

In the end it is important to remember that the lived experiences of victims can only be understood in light of their important intersections [55], such as religious affiliation among others. As the above review indicated, this is especially true with Muslim populations. Beyond research a continuous development and practice of cultural competence is an ethical obligation for practitioners in the field combating IPV [55]. Advocates who work with vulnerable IPV victims also have a special obligation to culturally competent service delivery especially when one considers how leaving abuse is difficult in any circumstance without added barriers and layers of complexity. This study is only a start to better understanding these complex dynamics and provides value by looking at a larger global context.

\section{Limitations}

The conclusions offered must also take into consideration several study limitations. First given the scope of this paper the study was narrowed down to all forms of intimate partner violence between married and/or adult intimate partners. While all forms of abuse were included, this study focus was not on family violence and did not include child abuse and abuse conducted by extended family. This study only looked at abuse of extended family if it was studied as part of IPV, and not if it was targeted as the primary focus. Therefore several studies that looked at honor killing were excluded. Additionally, this study is limited in that it only accessed articles written in English. Authors understand this does great disservice to studies on the topic conducted in other languages such as, Arabic, French, Urdu and Hindi for example. The authors also used their own tools for quality assurances for study sample methods and this can also pose a limitation. Selective reporting by published articles and reports with expected outcomes can also be a limitation in the sample of this study. However, the authors contend that, despite the limitations in this study, it still helps to identify the themes of previous studies, provides an overview, identifies limitations and gaps left by past studies, and informs future researchers regarding what directions to focus.

\section{References}

1. World Health Organization (2006) World report on violence and health. Geneva: WHO Technical Report Series.

2. Krug EG, Mercy JA, Dahlberg LL, Zwi AB (2002) The world report on violence and health. Lancet 360: 1083-1088.

3. Trevillion K, Oram S, Feder G, Howard LM (2012) Experiences of domestic violence and mental disorders: a systematic review and meta-analysis. PLoS One 7: e51740.

4. Anderson KM, Renner LM, Danis FS (2012) Recovery: resilience and growth in the aftermath of domestic violence. Violence Against Women 18: 1279-1299.

5. Howard LM, Feder G, Agnew-Davies R (2013) Domestic violence and mental health: Royal College of Psychiatrists Publications.

6. Fortune MM (2009) The Bible Says.

7. Ammar $\mathrm{NH}$ (2007) Wife battery in Islam: a comprehensive understanding of interpretations. Violence Against Women 13: 516-526.

8. Hassouneh-Phillips D (2003) Strength and vulnerability: spirituality in abused American Muslim women's lives. Issues Ment Health Nurs 24: 681-694.

9. Quraishi A, Syeed-Miller N (2004) No altars: A survey of Islamic family law in the United States. In L. Welchman (Ed.), Women's Rights and Islamic Family Law: Perspectives on Reform. Zed Books, New York, USA.

10. Alkhateeb MB, Abugideiri SE (2007) Change from within: Diverse perspectives on domestic violence in Muslim communities. Great Falls.

11. Alogali A, Jayasundara J (2013) Violence Against Women: Experiences in Saudi Arabia, The18th Biennial International Consortium for Social Development, Kampala, Uganda

12. Texas Muslim Women's Foundation, Inc. (2012) Peace in the home. Training on Domestic Violence in the Muslim Community to Collin County Coalition on Domestic Violence.

13. Alwani Z, Abugideiri S (2003) What Islam Says About Domestic Violence: A Guide to Helping Muslim Families. Herndon, USA.

14. Alkhateeb S, Ellis, S, Fortune M (2001). Domestic violence: The response of Christian and Muslim communities. Journal of Religion \& Abuse 2: 3-24

15. Abugideiri SE (2007) A perspective on domestic violence in the Muslim community. In M.B. Alkhateeb\& S.E. Abugideiri (Eds.), Change from within: Diverse perspectives on domestic violence in Muslim communities. Great Falls.

16. Hassouneh-Phillips D (2001c) Polygamy and wife abuse: A qualitative study of Muslim women in America. Health Care for Women International, 22: 735-748.

17. Abu-Ras W (2003) Barriers to services for battered Arab immigrant women in a Detroit suburb. Social Work Research and Evaluation 3: 49-66.

18. Abu-Ras W (2007) Cultural beliefs and service utilization by battered Arab immigrant women. Violence Against Women 13: 1002-1028.

19. Hassouneh-Phillips D (2001) American Muslim women's experiences of leaving abusive relationships. Health Care Women Int 22: 415-432. 
Citation: Jayasundara D, Nedegaard R, Sharma B, Flanagan K (2014) Intimate Partner Violence in Muslim Communities. Arts Social Sci J S1: 003. doi: 10.4172/2151-6200.S1-003

20. Hassouneh-Phillips D (2001b) "Marriage is half of faith and the rest is fear Allah": Marriage and spousal abuse among American Muslims. Violence Against Women 7: 927

21. Gharaibeh M, Oweis A (2009) Why do Jordanian women stay in an abusive relationship: implications for health and social well-being. J NursScholarsh 41 : 376-384

22. Alkhateeb S (1999) Ending domestic violence in Muslim families. Journal of Religion \& Abuse, 1: 49-59.

23. Faizi N (2001) Domestic violence in the Muslim community. Texas Journal of Women \& the Law, 10: 209

24. Fortune M, Abugideiri S, Dratch M (2010) A commentary on religion and domestic violence.

25. PRISMA (2009). Preferred Reporting Items for Systematic Reviews and MetaAnalyses checklist

26. Zaza S, Wright-De Agüero LK, Briss PA, Truman BI, Hopkins DP, et al. (2000) Data collection instrument and procedure for systematic reviews in the Guide to Community Preventive Services. Task Force on Community Preventive Services. Am J Prev Med 18: 44-74.

27. Greer N, Mosser G, Logan G, Halaas GW (2000) A practical approach to evidence grading. JtComm J Quallmprov 26: 700-712.

28. Reynolds J, Kizito J, Ezumah N, Mangesho P, Allen E, et al. (2011) Quality assurance of qualitative research: a review of the discourse. Health Res Policy Syst 9: 43

29. Ghayyur T (2009) Domestic Violence Survey Analysis February-March 2009

30. Celik A, Sabri B (2011) 801 Muslims Speak Up to Stop Family Violence.

31. Adam N, Schewe P (2007) A multilevel framework exploring domestic violence against immigrant Indian and Pakistani women in the United States. Journal of Muslim Mental Health 2: 5-20.

32. Cwikel J, Lev-Wiesel R, Al-Krenawi A (2003) The Physical and Psychosocia Health of Bedouin Arab Women of the Negev Area of Israel The Impact of High Fertility and Pervasive Domestic Violence. Violence Against Women, 9 : 240-257.

33. Haddad LG, Shotar A, Younger JB, Alzyoud S, Bouhaidar CM (2011) Screening for domestic violence in Jordan: validation of an Arabic version of a domestic violence against women questionnaire. Int J Womens Health 3: 79-86.

34. Dalal K, Andrews J, Dawad S (2012) Contraception use and associations with intimate partner violence among women in Bangladesh. J BiosocSci 44: 83-94.

35. Rahman M, Sasagawa T, Fujii R, Tomizawa H, Makinoda S (2012) Intimate partner violence and unintended pregnancy among Bangladeshi women. J Interpers Violence 27: 2999-3015.

36. Koening M, Hossain MB, Ahmed S, Haaga J (1999) Individual and community determinants of domestic violence in rural Bangladesh. Paper presented at the 1999 Population Association of America Meetings, NY,USA.

37. Dalal K, Rahman F, Jansson B (2009) Wife abuse in rural Bangladesh. J BiosocSci 41: 561-573.
38. Keenan CK el-Hadad A, Balian SA (1998) Factors associated with domestic violence in low-income Lebanese families. Image J NursSch 30: 357-362.

39. Okenwa LE, Lawoko S, Jansson B (2009) Exposure to intimate partner violence amongst women of reproductive age in Lagos, Nigeria: Prevalence and predictors. Journal of Family Violence, 24: 517-530.

40. Yount KM, Li L (2010) Domestic violence against married women in Egypt. Sex Roles, 63: 332-347.

41. Btoush R, Haj-Yahia MM (2008) Attitudes of Jordanian society toward wife abuse. J Interpers Violence 23: 1531-1554.

42. Almosaed N (2004) Violence against women: A cross-cultural perspective. Journal of Muslim Minority Affairs, 24: 67-88

43. Haj-Yahia MM (2000) Wife abuse and battering in the sociocultural context of Arab society. Fam Process 39: 237-255.

44. Desai S, Haffajee Z (2011) Breaking the Silence: Reclaiming Qur'anic Interpretations as a Tool for Empowerment and Liberatory Praxis for Dealing with Domestic Violence in Canadian Muslim Communities. Canadian Woman Studies 29: 1-2.

45. Kulwicki A, Hymes R, Hammad A, Killawi, A, Farrag, M (2010) Development of a Domestic Violence Risk Assessment Tool for Arab American Clients. ACCESS Health 6: 12-22.

46. Zakar R, Zakar MZ, Krämer A (2012) Voices of strength and struggle: women's coping strategies against spousal violence in Pakistan. J Interpers Violence 27: 3268-3298.

47. Ali A, Toner BB (2001) Self-esteem as a predictor of attitudes toward wife abuse among Muslim women and men in Canada. J SocPsychol 141: 23-30.

48. Haj-Yahia MM (2003) Beliefs about wife beating among Arab men from Israel: The influence of their patriarchal ideology. Journal of Family Violence 18: 193-206.

49. Haj-Yahia MM,Uysal A (2008) Beliefs about wife beating among medical students from Turkey. Journal of Family Violence 23: 119-133.

50. Haj-Yahia MM, Wilson RM, Naqvi SA (2012) Justification, perception of severity and harm, and criminalization of wife abuse in the Palestinian society. J Interpers Violence 27: 1932-1958.

51. Kulwicki AD, Miller J (1999) Domestic violence in the Arab American population Transforming environmental conditions through community education. Issues in Mental Health Nursing 20: 199-215.

52. Haj-Yahia MM (2002) Attitudes of Arab women toward different patterns of coping with wife abuse. Journal of Interpersonal Violence, 17: 721-745.

53. Ammar NH (2000) Simplistic stereotyping and complex reality of Arab-American immigrant identity: Consequences and future strategies in policing wife battery. Islam \& Christian-Muslim Relations 11: 51-70.

54. Snajdr E (2005) Gender, power, and the performance of justice: Muslim women's responses to domestic violence in Kazakhstan. American Ethnologist 32: 294-311.

55. Lockhart LL, Danis FS (2010) Domestic Violence: Intersectionality and Culturally Competent Practice. New York: Columbia University Press, USA.
This article was originally published in a special issue, Violence Against Immigrant Women: A Global Perspective Throughout the Life-Cycle handled by Editors. Nawal H. Ammar, Arshia U. Zaidi, University of Ontario Institute of Technology (UOIT), Canada 